\title{
Electron Ptychography Using Fast Binary 4D STEM Data
}

Colum M. O’Leary ${ }^{1}$, Emanuela Liberti ${ }^{1,2}$, Sean M. Collins ${ }^{3}$, Duncan N. Johnstone ${ }^{3}$, Mathias Rothmann ${ }^{1}$, Jingwei $\mathrm{Hou}^{3}$, Christopher S. Allen ${ }^{1,2}$, Judy S. Kim ${ }^{1,2}$, Thomas D. Bennett ${ }^{3}$, Paul A. Midgley ${ }^{3}$, Angus I. Kirkland $^{1,2}$ and Peter D. Nellist ${ }^{1}$

1. Department of Materials, University of Oxford, Oxford, UK.

2. electron Physical Sciences Imaging Centre (ePSIC), Diamond Light Source, UK.

3. Department of Materials Science and Metallurgy, University of Cambridge, Cambridge, UK.

The advent of fast pixelated detectors (FPDs) in scanning transmission electron microscopy (STEM) has provided a wealth of possibilities for nanoscale characterization, particularly in the field of electron ptychography. Modern low-noise integrating CMOS and electron counting detectors can record a high signal convergent beam electron diffraction (CBED) pattern at each probe position in a raster scan [1-2]. Many FPDs possess a high dynamic range, enabling the acquisition of bright-field and dark-field signals from the data set without saturation. Such dynamic ranges are essential for simultaneous high-angle annular dark-field (HAADF) imaging and focused-probe ptychography [3].

The super-resolution and low-dose capabilities of electron ptychography using FPDs have seen many recent developments [4], but slow dwell times are ultimately a severe limitation (i.e. $10^{-3} \mathrm{~s}$ vs $10^{-5} \mathrm{~s}$ for $\mathrm{ADF}$ imaging). It is possible, however, to increase the frame rates in certain electron counting detectors by decreasing the usable dynamic range. With decreasing dynamic range, both the cumulative dose (electrons per unit area of sample) for a given beam current, and scan distortions are significantly reduced, creating a route to low-dose electron ptychography. In this contribution, we explore the capabilities of binary (1-bit) ptychography in STEM as a low-dose phase-reconstruction technique using a standard monolayer of $\mathrm{MoS}_{2}$ as a test sample.

Figure 1(a) shows an example CBED pattern from one of $512 \times 512$ probe positions recorded for a monolayer of $\mathrm{MoS}_{2}$. The data was recorded using a Medipix3 detector on a JEOL ARM300CF $(\mathrm{HT}=$ $80 \mathrm{kV}, \alpha=22.48 \mathrm{mrad}$, dose $\approx 10,000 \mathrm{e} \AA^{-2}$ ). The counter depth of the Medipix 3 was set to $2 x 1$-bit, with which it can record CBED patterns at a rate of $10 \mathrm{kHz}$. This is compared to 6-bit and 12-bit modes, where the frame rates are limited to $2 \mathrm{kHz}$ and $1 \mathrm{kHz}$, respectively. Figure $1(\mathrm{~b})$ displays the phase of the Fourier transform of the acquired data for one specific spatial frequency. This demonstrates that, even for low-dose CBED patterns with isolated electrons, the phase of the diffracted beams is still strongly expressed in the data.

Figure 2 shows the phase reconstruction obtained from the acquired 4D data set using ptychography. The reconstruction technique used was the single side-band (SSB) method, where a weak-phase object is assumed and the constructive interference between overlapping diffraction beams (as shown in Figure 1(b)) is integrated [5-6]. An inverse Fourier transform is then performed to reconstruct the sampleinduced electron phase shifts. Following reconstruction, the structure of the $\mathrm{MoS}_{2}$ monolayer is visible.

A comparison between SSB ptychography and other ptychographic techniques on binary CBEDs will be demonstrated and discussed. Finally, the application of low-dose binary ptychography to metal-organic frameworks (UiO-66) and other beam-sensitive materials will be presented. 
[1] H. Ryll et al, Proceedings of Microscopy \& Microanalysis (2014) p. 1122-1123.

[2] D. McGrouther et al, Proceedings of Microscopy \& Microanalysis (2015) p. 1595-1596.

[3] H. Yang et al, Nature Communications 7 (2016), p. 12532.

[4] Y. Jiang et al, Nature 559 (2018), p. 343-349.

[5] J. M. Rodenburg, B. C. McCallum and P. D. Nellist, Ultramicroscopy 48 (1993), p. 304-314.

[6] T. J. Pennycook et al, Ultramicroscopy 151 (2015), p. 160-167.

[7] The financial support of JEOL (UK) Ltd. and the EPSRC is gratefully acknowledged.

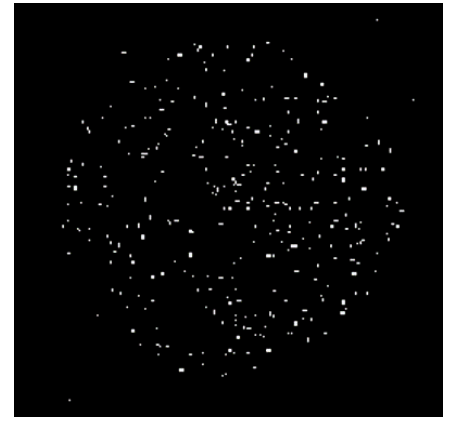

(a)

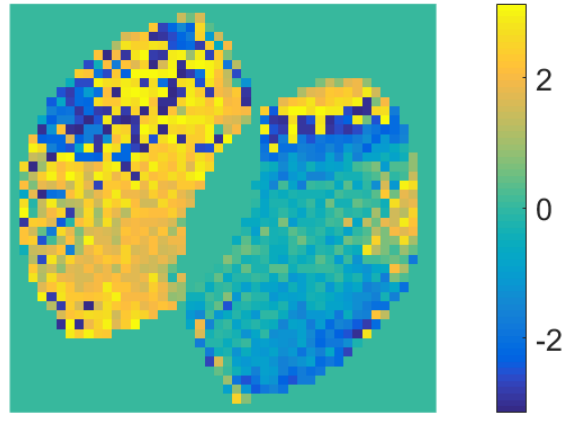

(b)

Figure 1. (a) Example of a binary convergent beam electron diffraction (CBED) pattern acquired for monolayer $\mathrm{MoS}_{2}$. (b) Phase of Fourier transform ('trotter') of the acquired 4D data set for a specific spatial frequency $(26 \mathrm{mrad})$. The trotter has been masked to show only regions of constructive interference. Color bar: phase (radians).
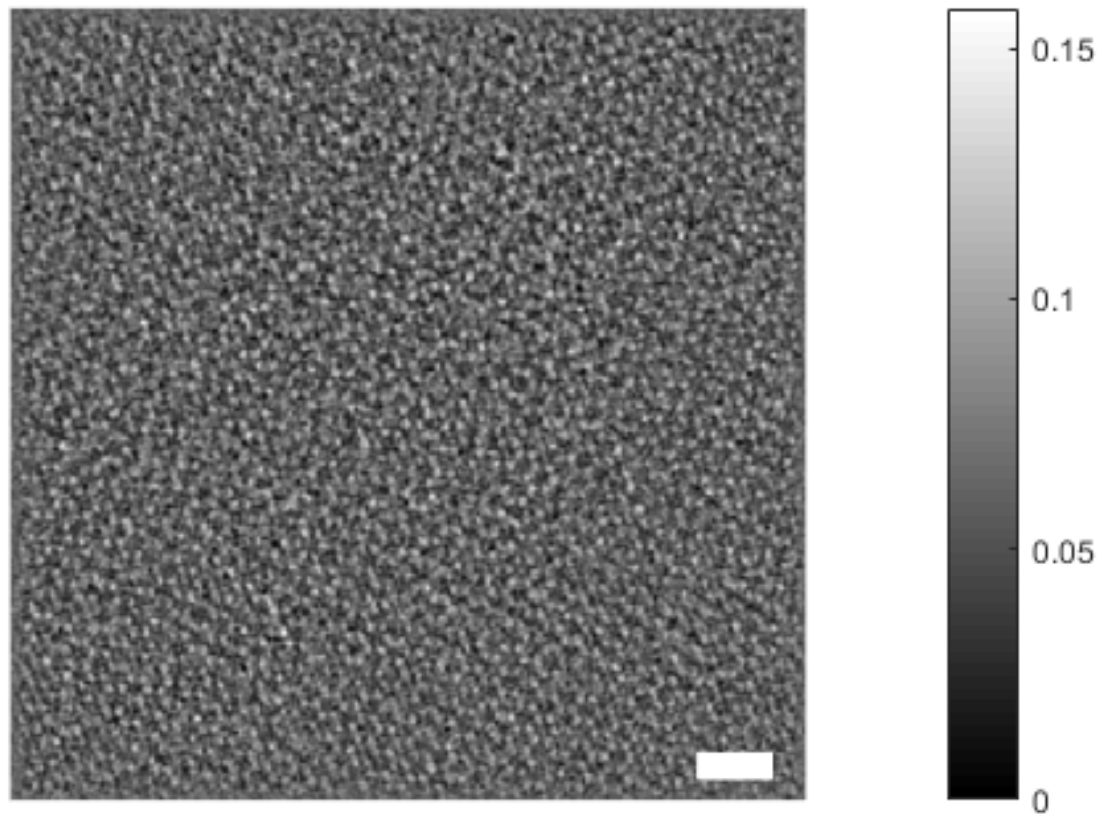

Figure 2. Ptychographic phase reconstruction of a monolayer of $\mathrm{MoS}_{2}$ using the single side-band method. Scale bar: $1 \mathrm{~nm}$. Color bar: phase (radians). 